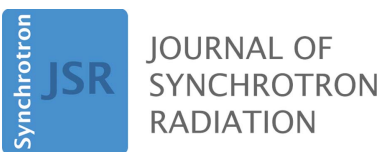

ISSN 1600-5775

Received 14 September 2018

Accepted 13 March 2019

Edited by I. Schlichting, Max Planck Institute for Medical Research, Germany

Keywords: footprinting; structural dynamics; beamline; solution state.

Supporting information: this article has supporting information at journals.iucr.org/s

\section{The XFP (17-BM) beamline for X-ray footprinting at NSLS-II}

\author{
Awuri Asuru, ${ }^{\mathrm{a}, \mathrm{b}}$ Erik R. Farquhar, ${ }^{\mathrm{a}}$ Michael Sullivan, ${ }^{\mathrm{a}}$ Donald Abel, ${ }^{\mathrm{a}}$ John Toomey, \\ Mark R. Chance $^{\mathrm{a} *}$ and Jen Bohon ${ }^{\mathrm{a} *}$ \\ ${ }^{a}$ Center for Synchrotron Bioscience, Department of Nutrition, Case Western Reserve University, \\ 10900 Euclid Avenue, Cleveland, OH 44106, USA, and ${ }^{\mathbf{b}}$ Systems Biology Graduate Program, Medical \\ Scientist Training Program, Case Western Reserve University, 10900 Euclid Avenue, Cleveland, OH 44106, \\ USA. *Correspondence e-mail: mrc16@case.edu, jbohon@bnl.gov
}

Hydroxyl-radical mediated synchrotron X-ray footprinting (XF) is a powerful solution-state technique in structural biology for the study of macromolecular structure and dynamics of proteins and nucleic acids, with several synchrotron resources available to serve the XF community worldwide. The XFP (Biological X-ray Footprinting) beamline at the NSLS-II was constructed on a three-pole wiggler source at $17-\mathrm{BM}$ to serve as the premier beamline for performing this technique, providing an unparalleled combination of high flux density broadband beam, flexibility in beam morphology, and sample handling capabilities specifically designed for XF experiments. The details of beamline design, beam measurements, and science commissioning results for a standard protein using the two distinct XFP endstations are presented here. XFP took first light in 2016 and is now available for general user operations through peer-reviewed proposals. Currently, beam sizes from $450 \mu \mathrm{m} \times 120 \mu \mathrm{m}$ to $2.7 \mathrm{~mm} \times 2.7 \mathrm{~mm}$ (FWHM) are available, with a flux of $1.6 \times 10^{16}$ photons s$^{-1}$ (measured at $325 \mathrm{~mA}$ ring current) in a broadband $(\sim 5-16 \mathrm{keV})$ beam. This flux is expected to rise to $2.5 \times 10^{16}$ photons s$^{-1}$ at the full NSLS-II design current of $500 \mathrm{~mA}$, providing an incident power density of $>500 \mathrm{~W} \mathrm{~mm}^{-2}$ at full focus.

\section{Introduction}

$\mathrm{X}$-ray footprinting (XF) mediated by the chemical activity of hydroxyl radicals was first demonstrated as a valuable tool for examining the structure and dynamics of biological macromolecules by studies of Brenowitz, Woodson, and Chance elucidating the folding mechanism of the Tetrahymena ribozyme over 20 years ago (Sclavi et al., 1997, 1998). Since that time, an array of XF approaches has explored a wide range of structural biology problems. XF of proteins (Kiselar et al., 2002), driven by the adoption of bottom-up mass spectrometry as the 'read-out', has now become a leading application of footprinting to understand the structure and dynamics of proteins. The technique provides a simple, direct, and rapid way to generate hydroxyl radicals in the solution phase, where the reactivity with protein side chains serves as an accurate probe of protein solvent accessibility. Favourable characteristics of hydroxyl radicals as footprinting reagents include: small size, solvent properties similar to water, and high reactivity with amino acid side chains. In addition, no reagents beyond those required for protein stability and functional poise are necessary for the experiment since the hydroxyl radicals are generated by water radiolysis.

Exposure of aqueous biological samples to ionizing radiation leads to the near instantaneous dissociation of water into water radicals, dry electrons, and excited waters. Within 
$10^{-12} \mathrm{~s}$, these initial products are converted into hydroxyl radicals and other primary radical species. The newly formed hydroxyl radicals diffuse through the solvent and rapidly react with the solvent-accessible side chains of amino acids, forming covalent oxidative modifications that can be identified and quantified using mass spectrometry (Takamoto \& Chance, 2006; Huang et al., 2015; Wang \& Chance, 2017). A number of oxidative modifications are possible upon reaction of hydroxyl radicals with protein side chains. The simplest mechanism for hydroxyl-mediated protein oxidation begins with $\mathrm{H}$ abstraction from a side chain carbon followed by reaction with $\mathrm{O}_{2}$ to form a peroxyl radical, which undergoes further reactions that lead to the formation of a hydroperoxide $(+32)$, hydroxide $(+16)$, or carbonyl $(+14)$ group. Other mechanisms such as insertion of hydroxyl radicals into aromatic rings (Phe, Trp, and $\mathrm{Tyr}$ ) or $\mathrm{H}$ abstraction from sulfhydryl groups (Cys or Met) are also possible. Detailed explanations of the various proposed mechanisms are beyond the scope of this paper. However, comprehensive explanations of the mechanisms and major products for each amino acid group are available in early reviews and papers on XF chemistry (Xu \& Chance, 2005, 2007).
Protein XF experiments consist of exposing controls (e.g. apo protein) and different experimental samples (e.g. ligandbound, complex, etc.) to an X-ray beam for varying exposure times. Downstream mass spectrometry (MS) analysis (Fig. 1) is conducted on each exposed sample and involves proteolytic digestion of X-ray exposed protein samples, separation of resultant peptides using ultra-performance liquid chromatography (UPLC), identification of modified and unmodified peptides using tandem mass spectrometry (MS/MS), and integration of extracted ion chromatogram (EIC) peaks to quantify the amount of unmodified and modified species for each peptide. The fraction of unmodified peptide is calculated for each exposure time and plotted to generate dose-response curves that are fit to single-exponential functions to determine first-order rate constants. Obtained rate constants can be compared across experimental conditions to monitor changes in solvent accessibility for the protein of interest in different functional and structural states.

By contrast, in nucleic acid (NA) footprinting experiments (Fig. 2), hydroxyl radicals cleave the NA phosphodiester backbone. Consequently, the relative accessibility of the NA backbone to cleavage is dependent on the conformation of the

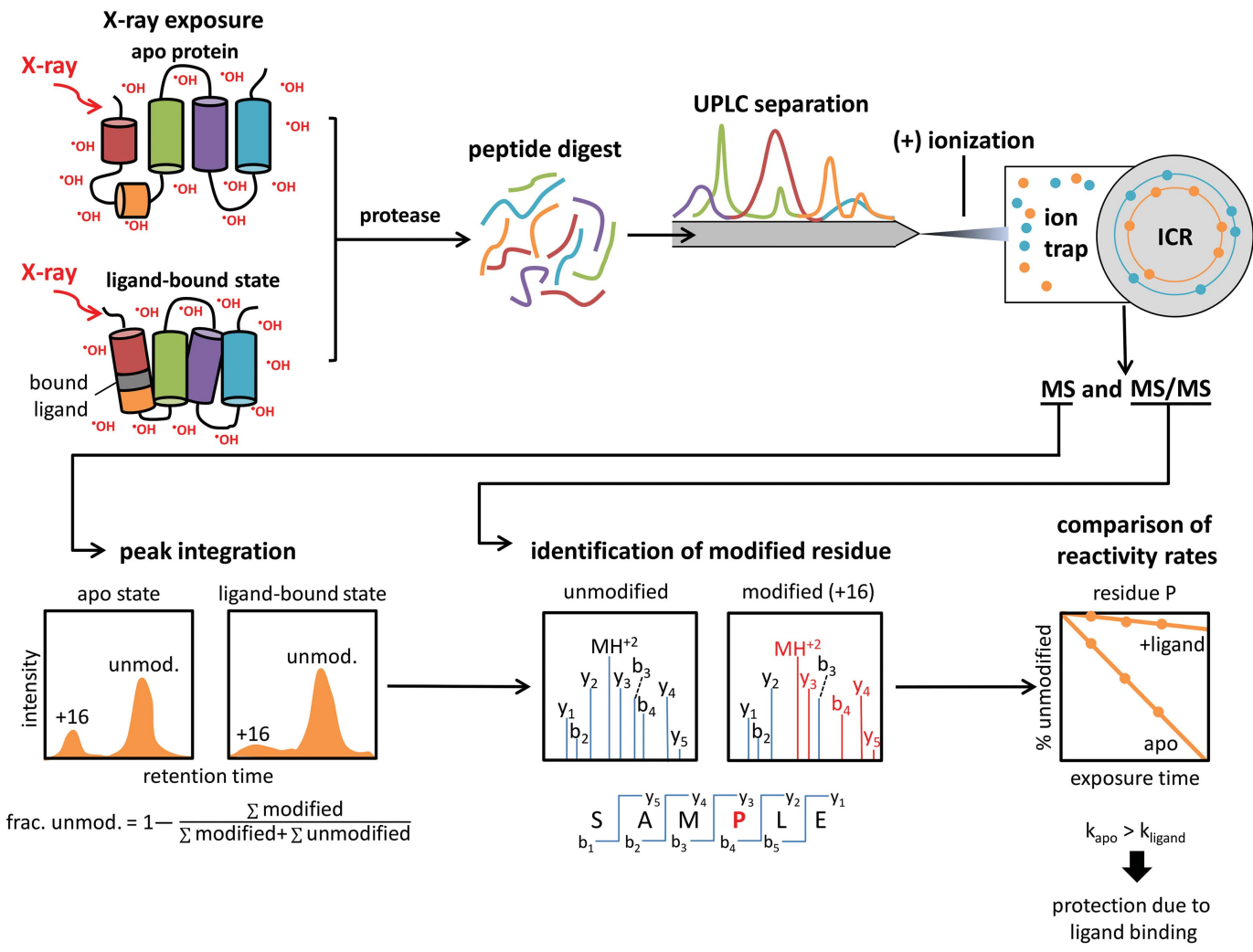

Figure 1

Schematic diagram of protein X-ray footprinting experiments. Control (e.g. apo) and experimental (e.g. ligand-bound) samples are exposed to an X-ray beam for microsecond to millisecond timescales. Exposed samples are digested with proteases to generate peptides, which are separated via ultraperformance liquid chromatography (UPLC). Electrospray ionization (ESI) is used to ionize the peptides, which enter the mass spectrometer where they are separated according to their mass-to-charge $(\mathrm{m} / \mathrm{z}$ ) ratios (MS) and are subsequently fragmented through collision with gas molecules (MS/MS). The fragmentation pattern is unique to each peptide, so MS/MS scans can be used to identify unmodified and modified peptides/residues within the sample. Extracted ion chromatograms (EICs) are used to quantify the amount of unmodified and modified peptides observed. The fraction of unmodified peptide remaining is calculated at each exposure time for each modified peptide/residue and plotted to produce dose-response curves, which are fit to a single-exponential function to determine first-order rate constants. The calculated rate constants in the control $\left(k_{\text {apo }}\right)$ and experimental $\left(k_{\text {ligand }}\right)$ samples are compared in order to determine regions of the protein that are important for function. 


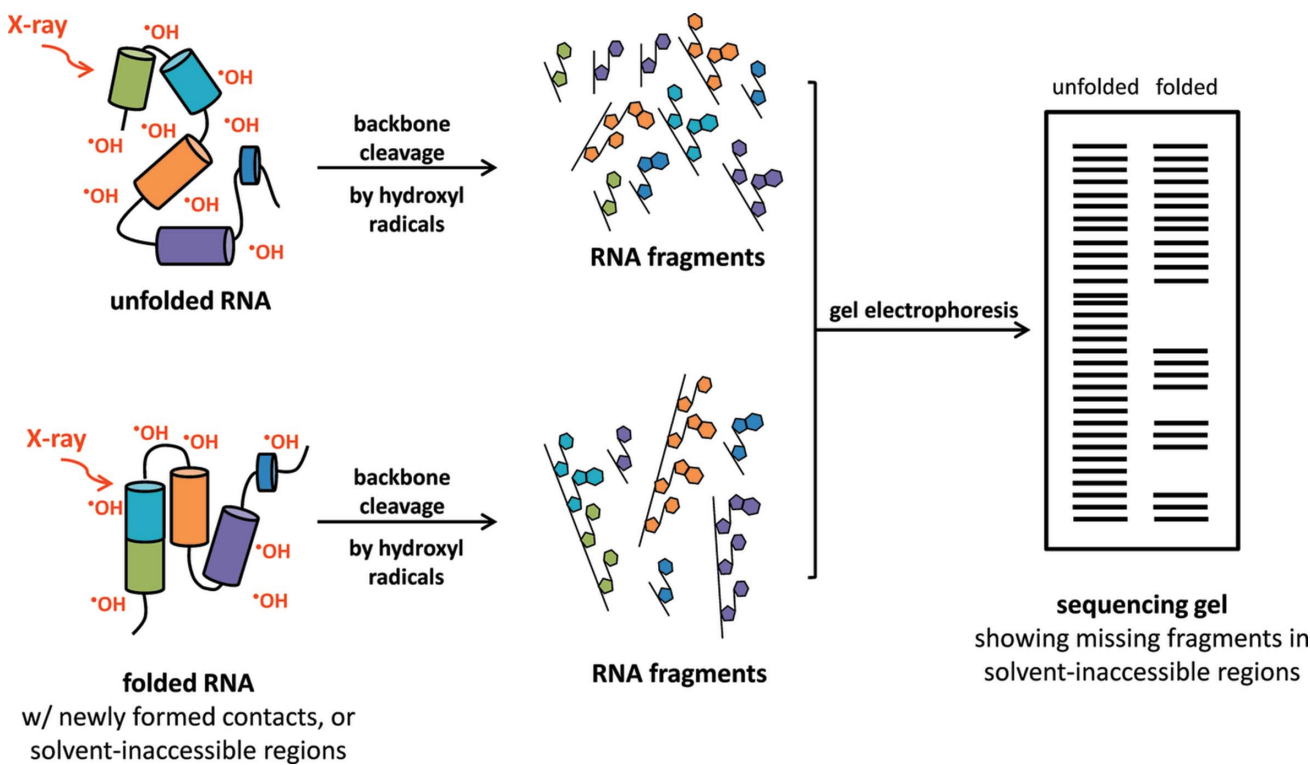

Figure 2

Schematic diagram of nucleic acid X-ray footprinting experiments. RNA folding experiments are an example application of X-ray footprinting. These experiments involve exposing unfolded and folded RNA samples to a focused X-ray beam, which produces hydroxyl radicals that cleave the phosphodiester backbone of the RNA molecule. The resulting nucleic acid fragments are analysed using gel electrophoresis. Missing regions observed on the sequencing gel for the folded sample indicate regions that become protected, or inaccessible to solvent (i.e. hydroxyl-radical cleavage), upon the unfolded RNA molecule assuming its folded state.

backbone and its engagement in inter- or intra-macromolecular interactions. The NA fragments are analyzed via gel electrophoresis or sequencing, and the resulting sequencing ladder is examined for regions that are not cleaved due to protection. This straightforward, X-ray-induced, hydroxylradical-mediated footprinting approach has been applied to a number of structural biology studies examining ligand/drug binding, epitope mapping of monoclonal antibodies, in vivo studies of nucleic acid footprinting, structural waters in proteins, and membrane proteins (Hulscher et al., 2016; Ralston et al., 2000; Gupta, Chai et al., 2014; Gupta et al., 2012; Kiselar et al., 2003; Adilakshmi et al., 2008; Clatterbuck Soper et al., 2013; Huang et al., 2018; Sangodkar et al., 2017).

These XF studies were performed at beamline X28C at NSLS and beamlines 5.3.1 or 3.2.1 at the Advanced Light Source (ALS). The XF beamlines at ALS are still available, but only 3.2.1, which has a considerably lower flux density than 5.3.1, is currently readily accessible to users. $\mathrm{X} 28 \mathrm{C}$ is no longer available due to the permanent shutdown of the NSLS in 2014. Construction of the NSLS-II has been completed, and the light source is capable of delivering X-rays four orders of magnitude brighter than the NSLS. The XFP (Biological X-ray Footprinting) beamline at NSLS-II $17-\mathrm{BM}$ is designed to harness the advanced capabilities of the new light source in order to produce flux densities greater than those previously available at X28C and other footprinting-capable beamlines. XFP will continue the footprinting program established by $\mathrm{X} 28 \mathrm{C}$, and significant effort is underway to develop new methodologies to study highly scavenging biological samples that have proven difficult to probe using previously available beamlines.
Complex biological systems such as macromolecular complexes, membrane proteins, and live cells often require high concentrations of various hydroxyl-radical-quenching reagents to preserve sample integrity, and therefore require greater radiation doses to overcome the effect of scavenging secondary reactions and to ensure sufficient radiolytic labelling of the protein sample (Gupta et al., 2007). The bulk of the hydroxyl radicals generated by water radiolysis last a few microseconds due to rapid self-reaction and reaction with 'targets' like amino acid side chains or nucleic acid bases; additionally, the nascent hydroxyl radicals can react with buffer reagents, molecular oxygen, and other solution components leading to scavenging of hydroxyl radicals and introduction of potential secondary reactions by propagated radicals. To accumulate the needed minimal dose (such that modified products are detected), a continuous radiation dose is maintained, providing a stable steady-state concentration of hydroxyl radicals throughout the footprinting experiment. This radiation dose may range from microseconds to milliseconds, depending on the beamline and sample characteristics, and must be increased for complex biological samples due to the presence of quenching reagents such as detergents, reducing agents, or stabilizing ligands (Gupta, Celestre et al., 2014; Bohon et al., 2014; Sullivan et al., 2008). Delivery of increased radiation dose is most easily accomplished by prolonging exposure of the sample to the X-ray beam. While increased exposure time is the simplest way to increase radiation dose, it is also the least optimal method due to damage or degradation of the protein sample caused by heat generation and secondary reactions mediated by superoxides, peroxides and other products of the radiolysis. Degradation of 
the sample also leads to chemical noise in LC-MS spectra, which makes it more difficult to accurately identify and quantify the observed modified and unmodified peptides. These issues can be partially circumvented by increasing the flux density of the beam, which increases the steady-state concentration of hydroxyl radicals without any need to extend exposure time (Gupta, Celestre et al., 2014).

This paper describes the technical details of the design and capabilities of the XFP beamline as well as measurements of beamline performance at two distinct endstations for XF. The capillary flow (CF) endstation allows for the exposure of samples to high X-ray doses on a microsecond timescale by quickly flowing samples through a narrow capillary exposed to the highly focused X-ray beam. The multi-sample holder (MSH) endstation is used to expose $5 \mu \mathrm{l}$ volumes of sample in PCR tubes and requires a wider, or lower flux density beam, to evenly expose stationary samples with full overlap. The CF endstation offers maximal X-ray dose delivery, while the MSH endstation offers speed and convenience since it allows for the exposure of up to 23 samples in the same amount of time ( $\sim 2-3 \mathrm{~min}$ ) required to expose just one sample using the $\mathrm{CF}$ endstation. Direct beam measurements were also taken to verify the flux, size and flux-density of the beam at XFP for $\mathrm{XF}$ experiments in the various endstation configurations, and benchmarking experiments with a model protein were also undertaken for both endstations. Based on these results, we anticipate the improved design of XFP will enable us to perform XF experiments that will allow significant advances in the field of hydroxyl radical footprinting of macromolecules.

\section{XFP beamline}

\subsection{Source and optics}

The XFP beamline is a pink beam beamline located at port 17-BM of NSLS-II on a three-pole wiggler (3PW) source that functions as a wavelength shifter $\left(E_{\text {crit }}=6.8 \mathrm{keV}\right)$ for NSLS-II bending-magnet radiation $\left(E_{\text {crit }}=2.39 \mathrm{keV}\right)$. The beamline accepts a $3 \mathrm{mrad}(\mathrm{H}) \times 0.33 \mathrm{mrad}(\mathrm{V})$ fan of radiation, focused using a $1.1 \mathrm{~m}$-long rhodium ( $\mathrm{Rh}$ )-coated toroidal mirror (Winlight $\mathrm{X}$ ) located in the front end of the beamline at $14 \mathrm{~m}$ from the source, downstream from a $250 \mu \mathrm{m}$-thick beryllium $(\mathrm{Be})$ window. The mirror substrate is single-crystal silicon ( $1.1 \mathrm{~m}$ long $\times 100 \mathrm{~mm}$ wide $\times 50 \mathrm{~mm}$ thick), with a cylindrical cut of sagittal radius $58.8 \mathrm{~mm}$ and a figure error of better than $1.5 \mu \mathrm{rad}$, designed specifically for XFP to provide 1:1 focusing at $28 \mathrm{~m}$ from the source. The chamber, kinematic mount and stainless steel bathtub with water cooling system were repurposed from NSLS X28C (Sullivan et al., 2008). Additional changes from the $\mathrm{X} 28 \mathrm{C}$ system include a new stand to accommodate the NSLS-II beam height, removal of the MACOR pucks, replacement of the eutectic bath with $2.98 \mathrm{~kg}$ of Indalloy 60 (removes tin from the system and increases the melting temperature of the eutectic), addition of absolute linear encoders (Renishaw), and replacement of the motion control system with NSLS-II Delta Tau Geobrick LV motor controllers. The toroidal mirror is set to a nominal $4.2 \mathrm{mrad}$

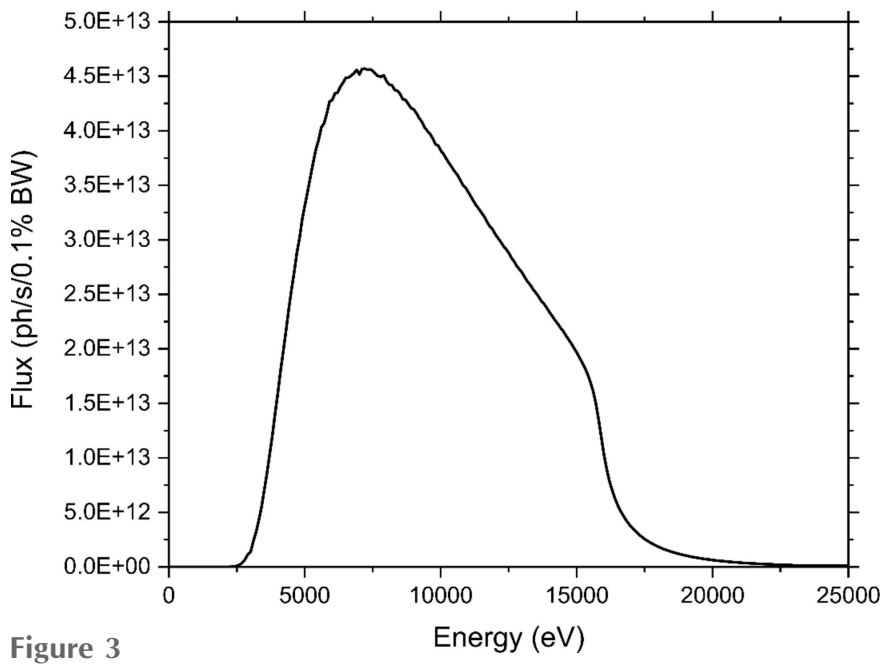

Spectral flux at XFP.

angle and is bendable to a minimum radius of $3 \mathrm{~km}$ to provide meridional focusing for adjustment of the vertical beam dimension. The horizontal beam size is determined by the sample distance from the focal point within the depth of the experimental enclosure. The beamline vacuum-air interface is a water-cooled $100 \mu \mathrm{m}$-thick diamond window with a $10 \mathrm{~mm}$ open aperture. This configuration delivers $>10^{16}$ photons s$^{-1}$ to the sample with a broadband energy range of $\sim 5-16 \mathrm{keV}$ and a peak flux near $7 \mathrm{keV}$. The resulting spectrum is shown in Fig. 3, calculated for the central $3 \mathrm{mrad}(\mathrm{H})$ and $0.33 \mathrm{mrad}(\mathrm{V})$ fan of the 3PW source using SRW software (Chubar et al., 2017); effects of beam optics were calculated using values from the Center for X-ray Optics (CXRO, http://henke. lbl.gov/optical_constants/).

\subsection{Photon delivery system}

In order to capture the maximum available radiation from the source, a significant portion of XFP beamline components are located inside the ratchet wall [Fig. 4(a)]. The first component after the crotch absorber is the bending-magnet photon shutter (BMPS), followed by the first and second fixed masks and the $250 \mu \mathrm{m}$-thick Be window that separates the ultra-high vacuum of the storage ring from the high vacuum of the beamline. Downstream of the Be window are the whitebeam slits (isolated for current measurement), the focusing mirror, two additional fixed masks, a water-cooled removable imaging screen, a photon shutter, final fixed mask, and a pair of safety shutters just upstream of the ratchet wall collimator. Along this path, two lead collimators, an inboard shadow shield, and a steel insert on top of the ratchet wall collimator allow the primary Bremsstrahlung radiation to be contained within the area inside the ratchet wall. Several gate valves are located along the beam path to allow maintenance of components; these valves are interlocked to close in the case of a vacuum leak. All beamline components downstream of the focusing mirror are shifted vertically to follow the $8.4 \mathrm{mrad}$ beam angle, and the exit port of the ratchet wall at $17-\mathrm{BM}$ is modified to accommodate the significantly shifted 
beam height. The photon delivery system downstream of the ratchet wall [Fig. 4(b)] includes approximately $3 \mathrm{~m}$ of permanent components after the gate valve, consisting of beam-defining pink beam slits (Oxford Danfysik, repurposed from NSLS X6B), a chamber for anticipated in-vacuum attenuators (in the design phase at the time of writing) and beam position monitor (Sydor Instruments, in commissioning at the time of writing), a pink beam photon shutter for sample protection, ion and turbo pumps, and vacuum isolation valves. In XF configurations, the downstream beam pipe is interchangeable to provide vacuum path to two endstations at different locations within the depth of the hutch, and vacuum pumping after the valve enables rapid return to $\sim 10^{-8}$ Torr; a water-cooled diamond exit window is transferrable to the end of any of these beam pipes to provide the vacuum-to-air interface.

2.2.1. Beam diagnostics. Beam diagnostics used at XFP include both commissioning and alignment tools. The first diagnostic in the beamline is integrated into the white-beam slits upstream of the focusing mirror; the blades are isolated for current measurement, allowing observation of changes in the position of the incoming photon white beam due to accelerator adjustments. To facilitate initial beamline commissioning, a water-cooled diagnostic screen (Axilon) is installed in the front end of XFP. The screen, pneumatically

A
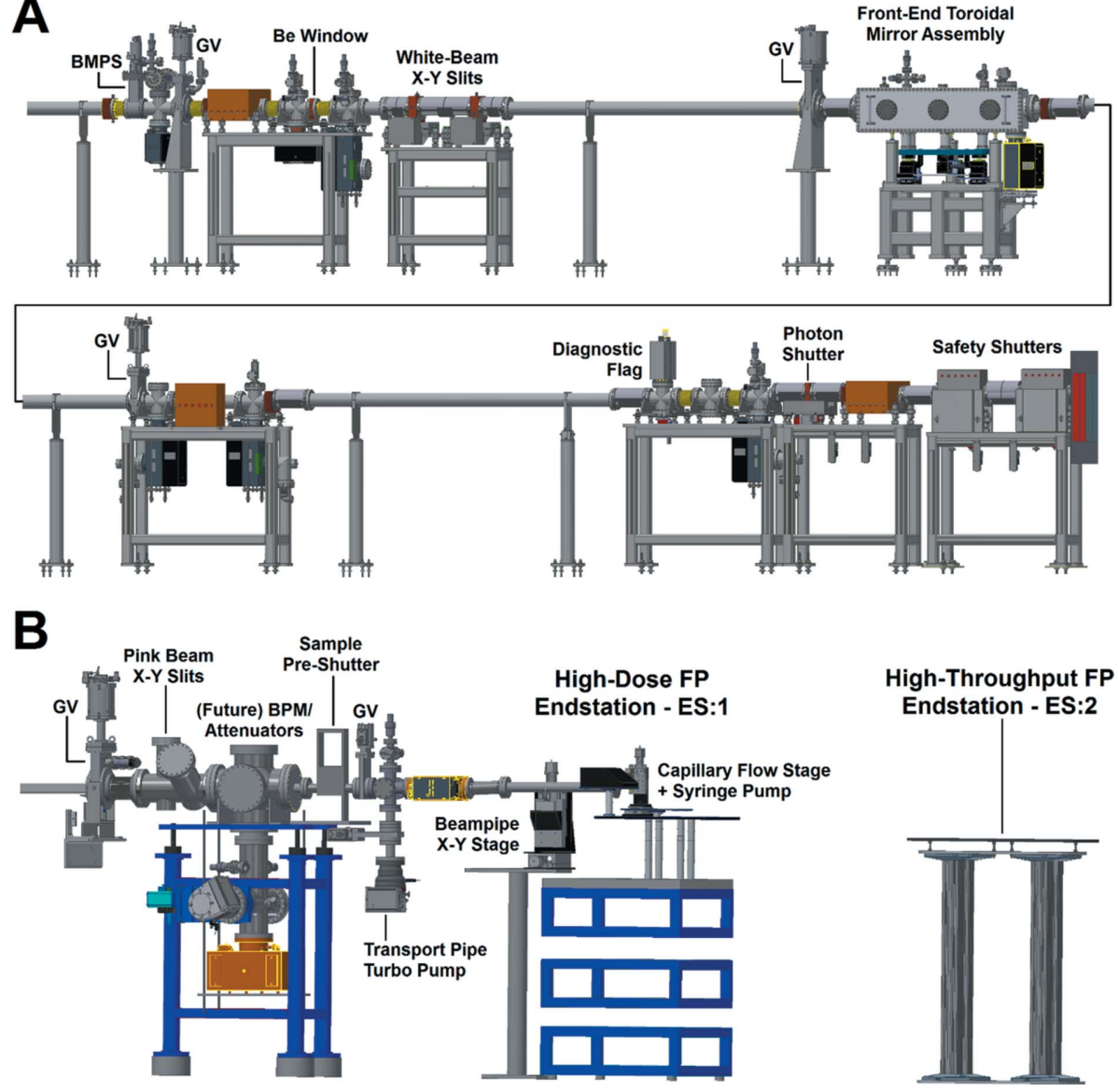

Figure 4

Elevation view of the XFP beamline, with the front-end components shown in panel A and the experimental hutch components shown in panel B.

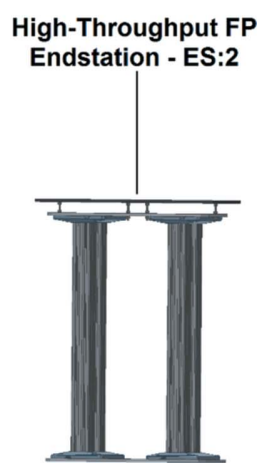

actuated to move in and out of the beam path, consists of a copper wedge coated with europium-doped $\mathrm{Y}_{2} \mathrm{O}_{3}(50 \mu \mathrm{m})$, tilted downward at $45^{\circ}$ with respect to the incoming beam for observation by a GigE machine vision camera (Prosilica) mounted on a viewport below. The screen saturates at beam currents higher than $\sim 10 \mathrm{~mA}$, but was critical in initial alignment of the toroidal focusing mirror to navigate the beam through the series of fixed masks in the front-end. Subsequent alignment and beam measurement diagnostics are utilized downstream in the experimental hutch. These are not permanently installed in the beamline, with the exception of a water-cooled diamond pink beam monitor immediately downstream of the pink beam slits that is currently being commissioned. These include single-channel and quadrant diamond detectors (Bohon et al., 2010), silicon diodes for use with attenuated beam, and a kilopixel diamond imaging detector for real-time beam morphology and flux measurement at the sample position during mirror adjustment to accommodate various sample requirements (Zhou et al., 2015).

\subsection{Experimental enclosure and endstations}

The experimental hutch enclosure $(\sim 9.7 \mathrm{~m} \times 2.5 \mathrm{~m})(\mathrm{GPS}$, Inc.) is located immediately downstream from the ratchet wall. It contains the last $3 \mathrm{~m}$ of the beamline and two experimental endstations that offer alternative modes of sample delivery, or exposure, to the X-ray beam [Fig. 4(b)]. The lead enclosure was designed according to NSLS-II standard specifications for use with white beam, with an additional lead beam stop added at the end of the enclosure to contain secondary radiation. Patch panels for BNC and Ethernet signals, as well as a user-accessible labyrinth, enable flexibility in adapting to experiment-specific user equipment. Gaseous nitrogen, continuously provided by the facility, is also available for use. Helium is piped in from compressed gas cylinders outside of the enclosure to enable use of a helium-filled flight tube for rapid change between XF endstations when required. A mount for aluminium attenuators to control X-ray dose is provided at the end of the vacuum flight path. Two cameras on floor-stand tripods with pan-tilt-zoom capabilities are moveable throughout the hutch for observation of ongoing experiments in real time. Brief descriptions of the capillary flow (CF) and multi-sample holder (MSH) endstations at XFP are provided in Sections 2.3.1 and 2.3.2. Further details and diagrams regarding 
setup and operation of these types of experimental endstations can be found in previously published beamline papers (Gupta et al., 2007; Gupta, Celestre et al., 2014; Bohon et al., 2014; Hulscher et al., 2016; Hao et al., 2018).

2.3.1. Capillary flow (CF) endstation (ES:1). The toroidal mirror and capillary flow (CF) experimental endstation are placed $14 \mathrm{~m}$ and $28 \mathrm{~m}$ from the source, respectively, resulting in a 1:1 focus within the experimental hutch. Studies involving large macromolecular complexes, live cells, isolated organelles, and highly scavenging buffers require higher flux density and are conducted using the CF endstation. Samples are exposed to an X-ray beam using a $200 \mu \mathrm{m}$ internal diameter (ID) capillary, allowing photons to be delivered to the sample over a small surface area. Apparatus for use at this endstation are mounted to a three-axis $x-y-z$ motorized optical table with 1/4-inch-20 tapped holes at 1-inch spacing. The capillary exposure cell, mainly comprising a copper block with attached stainless steel slits to guide the capillary, is watercooled to $4^{\circ} \mathrm{C}$ to assist in maintaining capillary integrity during exposure. The flow cell is mounted to an $x-y$ stage with precision slides to allow careful alignment of the capillary to the beam using a diamond imaging detector placed downstream of the apparatus. The capillary is mounted vertically such that the larger horizontal dimension of the beam fully covers the width of the capillary.

For standard CF experiments, samples are loaded into a $1 \mathrm{~mL}$ glass gas-tight syringe, and a high-pressure syringe pump (Harvard, PHD2000) is used to push the sample through a $200 \mu \mathrm{m}$ ID capillary exposed to the X-ray beam for varying amounts of time. Exposure time is a function of the flow rate of the sample, vertical beam size $(h)$, and capillary internal ID $(2 r)$. The capillary ID and vertical beam size are kept constant during a given experiment, so varying the flow rate of the sample is primarily used to control the exposure time. The following equation is used to calculate the required flow rate for a given exposure time,

Flow rate $[\mathrm{mL} / \mathrm{min}]=$

$$
\frac{\left(\pi r^{2}\left[\mathrm{~cm}^{2}\right] h[\mathrm{~cm}]\right) \times 60[\mathrm{~s} / \mathrm{min}] \times 10^{6}[\mu \mathrm{s} / \mathrm{s}]}{\text { exposure time }[\mu \mathrm{s}]} .
$$

Alternatively, the vertical beam size $\left(\right.$ e.g. $\left.120 \times 10^{-6} \mathrm{~m}\right)$ can be divided by the desired exposure time $\left(\right.$ e.g. $\left.150 \times 10^{-6} \mathrm{~s}\right)$ to calculate the required speed (e.g. $0.8 \mathrm{~m} \mathrm{~s}^{-1}$ ) of the sample. Immediately following exposure, samples are collected in microcentrifuge tubes placed at the end of the X-ray exposed capillary. The microcentrifuge tubes are prefilled with methionine amide, which is added to $10 \mathrm{~m} M$ target concentration, to ensure samples are quenched immediately after passing through the X-ray beam.

This endstation is also used to host the in vivo exposure apparatus. For this experiment, a circulating water bath set atop a stirrer, kept at $37^{\circ} \mathrm{C}$ using a Haake AC200 chiller (Thermo Scientific), is used as an incubator for liquid cell culture. A positive displacement pump (Vici M50) is used to control the rate of flow of cell culture through the capillary for exposure to the X-ray beam (Hao et al., 2018). A programmable fraction collector (Teledyne) is used to collect the much larger volumes of sample required (commonly $0.75 \mathrm{ml}$ per tube), and enable separation of time points in time-resolved experiments. The syringe pump used for the standard capillary flow experiments can be simultaneously controlled to serve to initialize a reaction (for example, to add food to starved cells). For live cell culture exposure, the capillary exposure apparatus is generally mounted beyond the focal point to obtain a horizontal beam dimension capable of fully covering the $530 \mu \mathrm{m}$ ID capillaries preferred for these experiments.

2.3.2. Multi-sample holder (MSH) endstation (ES:2). XF experiments involving the exposure of small, soluble proteins in non-scavenging buffers can be exposed using far less flux density. These experiments are executed using the multisample holder (MSH) endstation, which allows 23 samples to be exposed in $\sim 2-3 \mathrm{~min}$. Sample droplets $(5 \mu \mathrm{L})$ are exposed in $200 \mu \mathrm{L} \mathrm{PCR} \mathrm{(polymerase} \mathrm{chain} \mathrm{reaction)} \mathrm{tubes} \mathrm{(Brand-}$ tech/FisherScientific Catalogue number 1388258), which are designed for excellent thermal transfer. This mode of sample exposure requires a wider beam to ensure that the $\mathrm{X}$-ray beam spot completely covers the surface area of the sample droplet ( $\sim 2.5 \mathrm{~mm}$ diameter). Because the focusing mirror is set to a nominally fixed angle, the MSH experimental endstation is placed further back in the hutch ( $30 \mathrm{~m}$ from the source) in order to increase the horizontal beam width. The increased beam width reduces the flux density on the sample, and therefore millisecond exposure times (regulated by a fast shutter, see below) are necessary to deliver adequate X-ray dose to the sample. Despite the decreased flux density, MSH experiments are advantageous because they reduce the time and sample volume required for a given experiment.

For MSH experiments, PCR tubes containing sample aliquots are exposed sequentially by loading the tubes into the sample holder mounted to a precision motorized slide. An electronic Uniblitz (Vincent Associates) beam shutter controls exposure time, with a $10 \mathrm{~ms}$ minimum reliable exposure time. During exposure, the temperature of the sample holder is controlled according to the needs of a given experiment by a circulating water bath and Peltier coolers. The apparatus can be cooled to $-30^{\circ} \mathrm{C}$, which is required for maintaining nucleic acid integrity during frozen cell experiments (Adilakshmi et al., 2006) and enables water-protein interactions to be studied by exposing frozen solution samples (Gupta et al., 2012). After exposure, methionine amide is added to $10 \mathrm{~m} M$ target concentration in the exposed sample to quench any remaining hydroxyl radicals and prevent secondary reactions.

\subsection{Controls}

Motor systems at XFP are driven via Delta Tau Geobrick LV motor controllers, which are the standard controller adopted by NSLS-II. Beamline controls are implemented through EPICS (Experimental Physics and Industrial Control System), Ophyd/Bluesky (Arkilic et al., 2017), a Python-based 
data acquisition system developed by the NSLS-II Controls Group, and Control System Studio (CSS). EPICS input/output controllers (IOCs) are run on a server local to the beamline. CSS is used to access EPICS process variables (PVs), generally for beamline alignment, maintenance and troubleshooting. Ophyd translates the PVs from hardware output to a generally accessible unified program interface, which can then be used by Bluesky for a variety of experiment controls and data acquisition. Bluesky programs are used to run all endstations at XFP, with a graphical user interface for the $\mathrm{MSH}$, and command-line control of the capillary flow and in vivo exposure systems (GUIs planned in the future). All programs are freely available on GitHub (https:/github.com/ NSLS-II-XFP).

\subsection{Sample preparation facilities}

Samples for XF experiments have a variety of sample preparation requirements. Standard laboratory facilities (https://www.bnl.gov/ps/labs/) are available in attached Laboratory Office Buildings (741, 743, with 744 and 745 labs under construction); however, some samples require preparation closer to the beamline for various reasons (sample environment stability, biosafety level, short lifetime of intermediates, etc.). For these purposes, as well as convenience, a separate sample preparation area is located at the XFP beamline immediately downstream of the control station. This area contains several workbenches with excellent lighting, a cooling centrifuge for microcentrifuge tubes or PCR tubes, a microbalance, a UV/Vis spectrometer, a handheld fluorimeter for measurement of Alexa488 fluorescence, single and multi-channel pipettes, an incubator/shaker, and consumables required for use of this equipment. The area is relatively well separated from other beamlines at NSLS-II, which allows designation of the area (and the hutch) as acceptable for exposure of biosafety level 2 samples under controlled circumstances with appropriate preparation.

\section{Beamline performance}

XFP was designed to provide higher radiation doses on shorter time scales compared with currently and previously available XF beamlines, with flexibility in the beam size, shape, and power density delivered to the sample. NSLS X28C, the predecessor to XFP, had the potential to reach $90 \mathrm{~W} \mathrm{~mm}^{-2}$ in power density (Sullivan et al., 2008), but only utilized a maximum of $\sim 40 \mathrm{~W} \mathrm{~mm}{ }^{-2}$ due to sample handling limitations; the target performance goal for XFP is $500 \mathrm{~W} \mathrm{~mm}^{-2}$, for an order of magnitude increase over what was previously used at X28C. To obtain high quality XF data, it is critical to ensure that the dose is deposited as uniformly as possible throughout the sample. This requirement drove the choice of energy range for the beam $(\sim 5-16 \mathrm{keV})$, as a compromise between deposition characteristics of lower energy photons (minimal penetration) versus higher energy photons (minimal absorption) in the sample and the ability to generate radicals efficiently at sample depths of up to several millimeters. Aluminium attenuators (typically $50-1000 \mu \mathrm{m}$ ) are also used to moderate flux and change the energy spectrum of various experimental configurations. The need for a variety of available beam shapes to accommodate different sample morphologies is also apparent from the above discussion. Three such sample configurations are currently available at XFP: capillaries of 200 and $530 \mu \mathrm{m}$ ID (for standard capillary flow and in vivo experiments, respectively), and a $5 \mu \mathrm{l}$ sample held by surface tension at the bottom of a PCR tube (for MSH experiments), with a sample diameter of $\sim 2.5 \mathrm{~mm}$. Beam measurements were made at each of these configurations, and standard samples were exposed in both capillaries and PCR tubes to verify beamline performance.

\subsection{Beam shape, size, and power}

A pixelated transmission-mode diamond detector was used to monitor the beam position, shape, and flux of XFP during real-time adjustment of the toroidal focusing mirror to obtain beam shapes appropriate for capillary flow and in vivo $\mathrm{XF}$ experiments (Fig. 5, left and center, respectively). Construc-
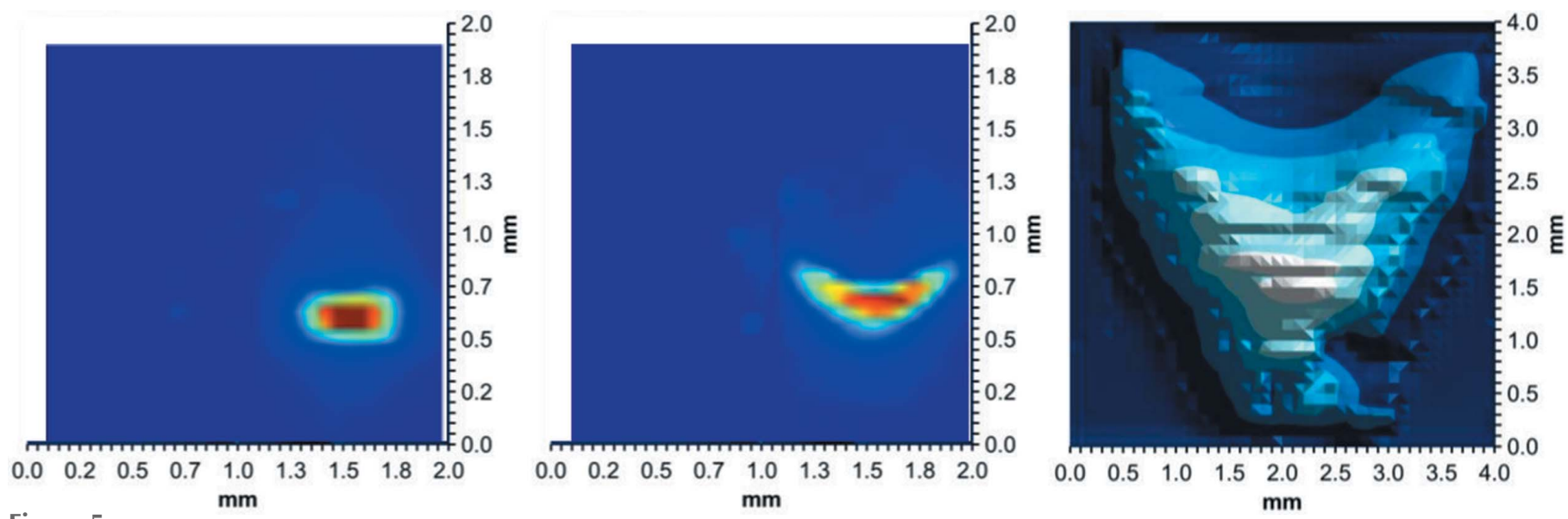

Figure 5

Beam profiles for use of various endstations at XFP. Left: focused beam at $\sim 28 \mathrm{~m}$ from the source $(120 \mu \mathrm{m} \times 450 \mu \mathrm{m}$ FWHM) for the $200 \mu \mathrm{m}$ capillary flow apparatus. Center: focused beam $(120 \mu \mathrm{m} \times 750 \mu \mathrm{m}$ FWHM) for use with $530 \mu \mathrm{m}$ capillaries for in vivo flow experiments. Right: unfocused beam $(2.7 \mathrm{~mm} \times 2.7 \mathrm{~mm}$ FWHM) for high-throughput experiments. 
tion and operation of the imaging detector is described elsewhere (Zhou et al., 2015). To determine appropriate mirror focusing parameters for the MSH endstation, the beam required is larger than this detector, thus the beam profile (Fig. 5, right) was measured by scanning a $100 \mu \mathrm{m}$ pinhole through the beam with a single channel detector behind the pinhole. Initial real-time adjustment of the mirror for this much larger beam was performed using a camera and fluorescent screen (copper block coated with $50 \mu \mathrm{m}$ europiumdoped $\mathrm{Y}_{2} \mathrm{O}_{3}$ ). For precision measurements of the beam size, quadrant single-crystal diamond detectors were scanned across the beam; these detectors are capable of measurement to a precision of $0.1 \%$ of the beam size. Measurements of the beam power were performed using both diamond detectors and via copper block calorimetry. The highest power density beam directly measured at the time of writing was $290 \mathrm{~W} \mathrm{~mm}^{-2}$, with $15.7 \mathrm{~W}$ in a beam size of $450 \mu \mathrm{m}(\mathrm{H}) \times$ $120 \mu \mathrm{m}(\mathrm{V})$ (FWHM). The measurement was made at a ring current of $325 \mathrm{~mA}, 210 \mathrm{~mm}$ from the exit window and inside of a nitrogen-filled enclosure to protect the detector from ozone corrosion. Standard CF sample positioning is less than $100 \mathrm{~mm}$ from the exit window; the estimated corresponding power density delivered to the actual sample position is thus $345 \mathrm{~W} \mathrm{~mm}^{-2}$ (calculated using the XFP spectrum and optical constants obtained from CXRO), with a total flux of $1.6 \times$ $10^{16}$ photons s$^{-1}$ based on the spectrum (Fig. 3). When NSLSII achieves the design goal of $500 \mathrm{~mA}$ beam current, XFP is thus expected to be capable of delivering up to $>500 \mathrm{~W} \mathrm{~mm}^{-2}$ to the focal position at $28 \mathrm{~m}$ from the source, with a total flux of $\sim 2.5 \times 10^{16}$ photons $^{-1}$. This power density is $>5$-fold greater than was previously possible (and $>10$-fold greater than was employed) at X28C (Sullivan et al., 2008; Bohon et al., 2014), and meets the design goals of the beamline.

\subsection{Alexa 488 fluorophore measurement}

To further evaluate beamline capability, the degradation rate of Alexa 488 fluorophore was used as a proxy for radiation dose in the sample. With increased exposure to an X-ray beam, Alexa fluorescence decreases with first-order kinetics, so calculated rates for Alexa 488 degradation can serve as a relative measurement of radiolytic dose (Gupta et al., 2007). All Alexa dose-response experiments are performed at room temperature due to the temperature sensitivity of the dye, and exposed samples are immediately analysed following exposure using a Turner Biosystems TBS-380 fluorometer. The fraction of remaining fluorescence is calculated and plotted versus exposure time, and the resulting rate constant is used to compare the efficiency of hydroxyl radical formation under the different experimental conditions.

The maximum rate $\left(67000 \mathrm{~s}^{-1}\right)$ measured for Alexa 488 fluorophore exposed using high-speed capillary flow at XFP was 229-fold greater than the rate published for SOLEIL, 33fold higher than the highest rate published for NSLS X28C, and 4.9 -fold greater than the maximum rate published for beamline 5.3.1 at ALS (Table S1 of the supporting information) (Gupta et al., 2007, 2014; Baud et al., 2017). It should be

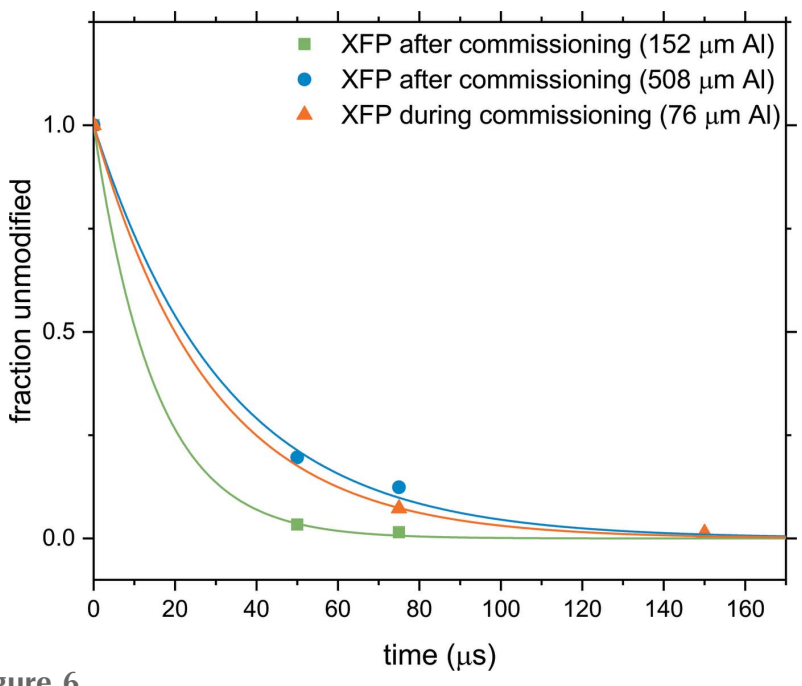

Figure 6

Dose-response curves for NSLS-II XFP at optimal beam focus based on Alexa 488 fluorescence decay in $10 \mathrm{~m} M$ sodium phosphate buffer ( $\mathrm{pH}$ 7.4) upon X-ray exposure using a high-speed capillary flow (CF) system. During commissioning, a rate of $35000 \mathrm{~s}^{-1}$ was obtained with $76 \mu \mathrm{m} \mathrm{Al}$ attenuation and a beam current of $250 \mathrm{~mA}$. Alexa studies completed after commissioning at $400 \mathrm{~mA}$ ring current resulted in rates of $67000 \mathrm{~s}^{-1}$ and $31000 \mathrm{~s}^{-1}$ using $152 \mu \mathrm{m} \mathrm{Al}$ and $508 \mu \mathrm{m} \mathrm{Al}$, respectively.

noted that this value was attained using a $152 \mu \mathrm{m}$ aluminium (Al) attenuator, while the analogous measurements at other beamlines were conducted in air or vacuum. Thus, the comparable un-attenuated rates for XFP using this assay would be considerably higher. Fig. 6 shows the dose-response curves for Alexa 488 decay fluorescence both during and after commissioning of XFP. Measuring maximal Alexa fluorescence decay rates is challenging on high-flux beamlines (e.g. NSLS-II XFP, NSLS X28C, ALS 5.3.1, etc.) because they produce high steady-state concentrations of hydroxyl radicals on short timescales, leading to near total quenching of Alexa 488 fluorescence within the first few exposure time points. As seen here, attenuation can be used to bring the dose into range. Given the $500 \mathrm{~mA}$ design beam current for NSLS-II, alternative improved hydroxyl radical dosimetry assays will need to be pursued for high-dose XF experiments.

\subsection{X-ray footprinting of cytochrome $\mathrm{c}$ as a standard sample}

Cytochrome $c$ is an excellent benchmark sample because it is a small, soluble protein with a well characterized structure and function, which has successfully been used to assess the capabilities of beamlines at the APS (10-BM-A), ALS (8.3.2, 5.0.2, and 5.3.1), CHESS (A2), and, most recently, NSLS (X28C), NSLS-II (17-BM) (Bohon et al., 2014). Similar proteins (e.g. ubiquitin, myoglobin, etc.) can serve as equally effective benchmark samples, but cytochrome $c$ has been chosen for beamline scientific commissioning because it allows accumulation of historical data that simplifies mass spectrometry data analysis and comparison of beamline performance.

3.3.1. Cytochrome c irradiation. Rabbit cytochrome $c$ (UniprotKB P00008) was purchased from Sigma-Aldrich and diluted in phosphate-buffered saline (PBS; $1 \mathrm{mM} \mathrm{KH}_{2} \mathrm{PO}_{4}$, $3 \mathrm{~m} M \mathrm{Na}_{2} \mathrm{PO}_{4}, 155 \mathrm{mM} \mathrm{NaCl}, \mathrm{pH}$ 7.4) (ThermoScientific) 
to a final concentration of $5 \mu M$. For MSH experiments, $5 \mu \mathrm{L}$ aliquots were exposed for 10-30 ms at room temperature and were pooled to collect a total of $25 \mu \mathrm{L}$ for each exposure time. The X-ray beam used for MSH experiments was $>2.5 \mathrm{~mm}$ in diameter and was attenuated with $762 \mu \mathrm{m}$ of $\mathrm{Al}$, with an NSLS-II storage ring current of $300 \mathrm{~mA}$. For CF experiments, $100 \mu \mathrm{L}$ of $5 \mu \mathrm{M}$ cytochrome $c$ was exposed for $75-300 \mu \mathrm{s}$. The size of the beam used for capillary flow experiments was $450 \mu \mathrm{m} \times 120 \mu \mathrm{m}$, with storage ring current of $250 \mathrm{~mA}$. The total X-ray dose of the beam was attenuated using $508 \mu \mathrm{m} \mathrm{Al}$. Immediately following exposure, samples were quenched with a final concentration of $10 \mathrm{~m} M$ methionine amide, flash frozen using liquid nitrogen, and stored at $-80^{\circ} \mathrm{C}$ until mass spectrometry analysis.

3.3.2. Mass spectrometry analysis. For all samples, $1 \mu \mathrm{g}$ of cytochrome $c$ was reduced and alkylated by incubation with $10 \mathrm{~m} M$ DTT for $1 \mathrm{~h}$ at $37^{\circ} \mathrm{C}$ followed by incubation with $25 \mathrm{~m} M$ iodoacetamide for $30 \mathrm{~min}$ at room temperature under dark conditions. After reduction and alkylation, the samples were digested with Lys- $\mathrm{C}$ for $3 \mathrm{~h}$ at $37^{\circ} \mathrm{C}$ followed by overnight trypsin digestion at $37^{\circ} \mathrm{C}$. Both proteases were used in a 1:10 $(w / w)$ enzyme:protein ratio. Peptides were analyzed by a ThermoScientific Orbitrap Elite hybrid ion trap mass spectrometer interfaced with a Waters nanoACQUITY UPLC according to standard procedures.

3.3.3. Data analysis. MS/MS spectra were manually validated using MassMatrix software (Xu \& Freitas, 2009). The
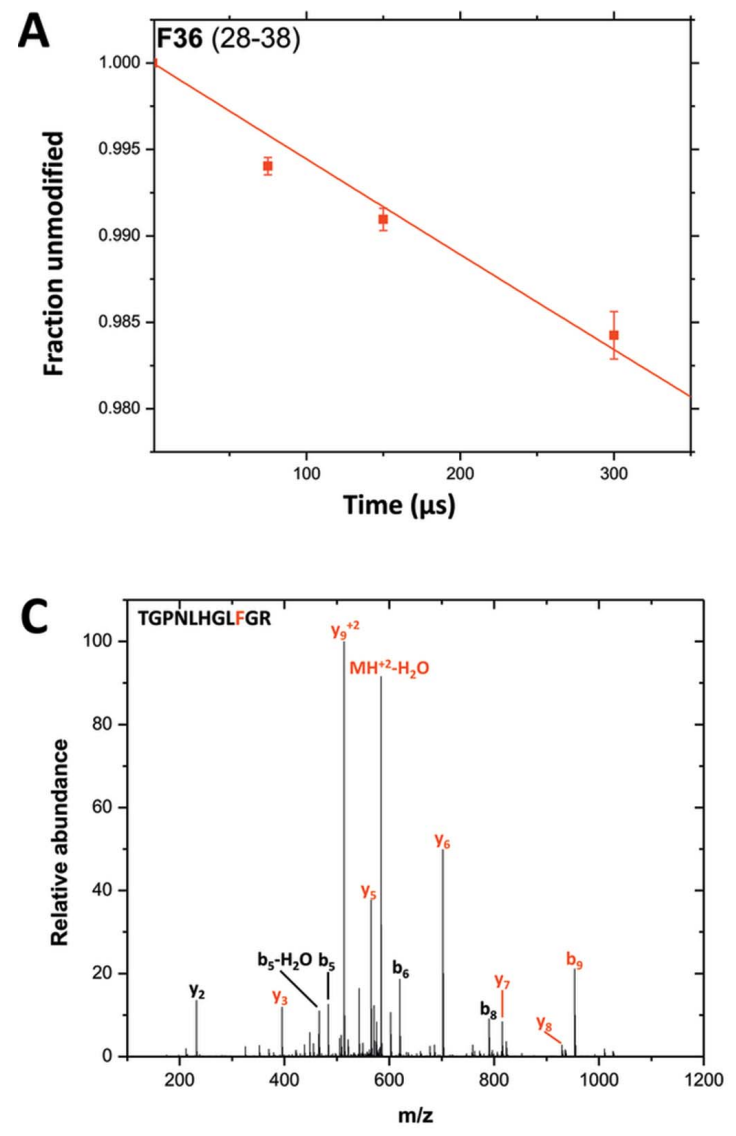

Figure 7

Example dose-response curves (A, B) and MS/MS scans (C, D) are shown for F36 in both the CF (A, C) and MSH (B, D) datasets. abundance of unmodified and oxidized species for each peptide was determined by extracting and integrating their selected ion chromatograms using XCalibur software from Thermo Fisher Scientific. The fraction of unmodified peptide for each exposure time was calculated based on previous approaches (Takamoto \& Chance, 2006). Dose-response curves were generated by plotting the fraction of unmodified peptides versus attenuation level, and the curves were fit to a single exponential equation using Origin 8 . The equation is described by

$$
y=\exp (-k t)
$$

where $k$ is the rate of modification in $\mathrm{s}^{-1}$ and $t$ is the exposure time in $\mathrm{s}$.

3.3.4. Results. MSH and CF data generated using XFP were compared with results from previous beamline experiments (Bohon et al., 2014). In previous benchmarking experiments, 11-13 modified residues were observed for cytochrome $c$, while, for experiments conducted on XFP, 11 and 14 modified residues (Fig. S1) were identified for cytochrome $c$ exposed using capillary flow and $\mathrm{MSH}$, respectively. $\mathrm{CF}$ and $\mathrm{MSH}$ benchmarking experiments both resulted in reproducible, radiolytic labelling of the sample, and all dose-response plots for both datasets are available in the supporting information (Figs. S2 and S3). Dose-response plots of the residue F36 are shown as an example [Figs. 7(A) and 7(B)]. The identity of F36 was confirmed via manual validation of $\mathrm{MS} / \mathrm{MS}$ scans from
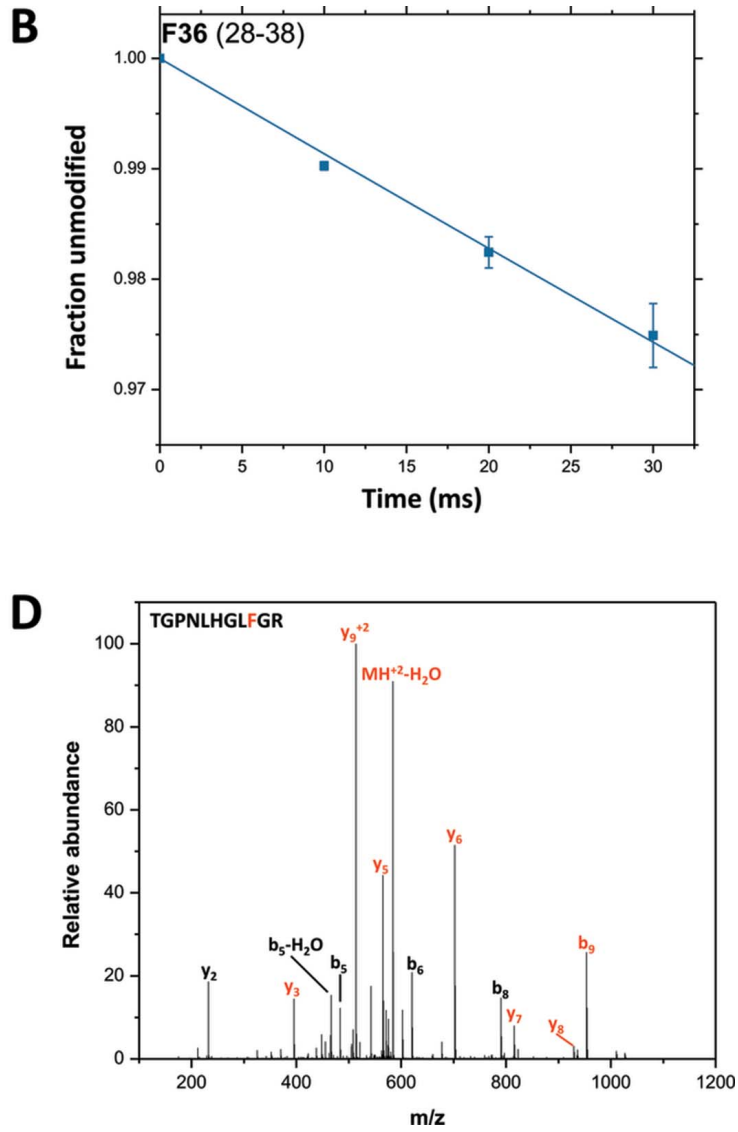

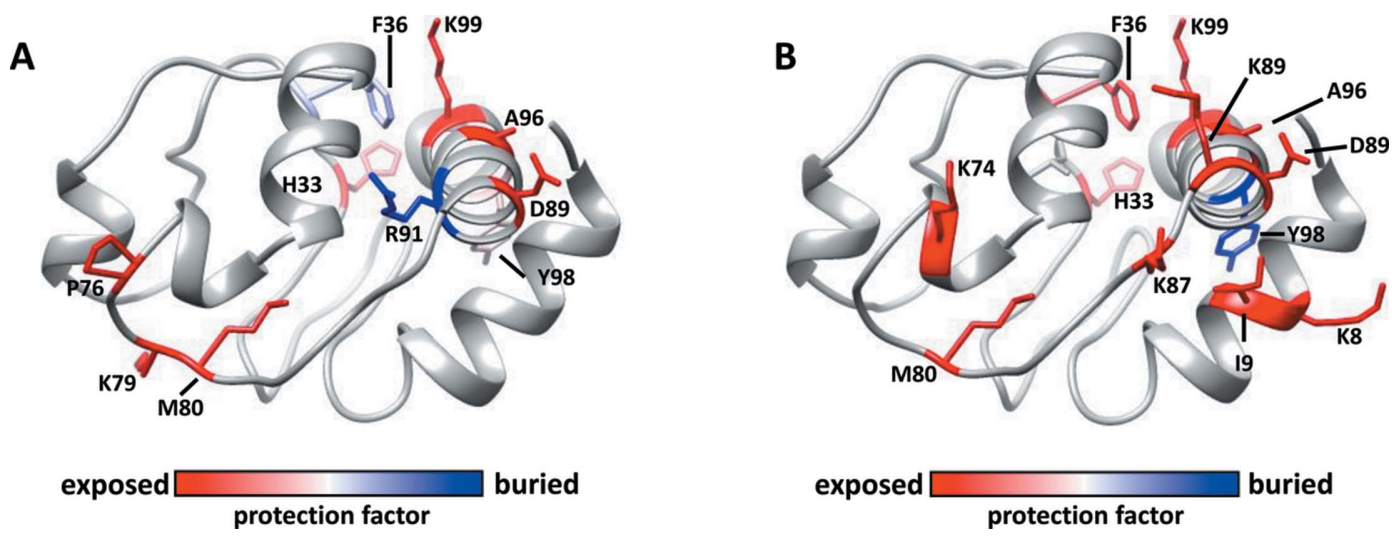

Figure 8

Calculated protection factor values mapped onto the structure of rabbit cytochrome $c$ are shown for F36 in both CF (A) and MSH (B) datasets.

each of the extracted ion chromatograms (EICs) belonging to the residue [Figs. 7(C) and 7(D)].

Signal-to-noise $(\mathrm{S} / \mathrm{N})$ ratios of the EICs were also examined in order to assess data quality. The $\mathrm{S} / \mathrm{N}$ ratios for the unmodified and modified extracted ion peaks for peptide 28-38 were determined for the $75 \mu \mathrm{s}$ and $30 \mathrm{~ms}$ exposures of the $\mathrm{CF}$ and $\mathrm{MSH}$ data, respectively. These exposure times were chosen for $\mathrm{S} / \mathrm{N}$ analysis because they had roughly equivalent $\mathrm{X}$-ray doses based on Alexa dose-response studies showing $\sim 38 \%$ fluorescence remaining following X-ray exposure (Fig. S4). The calculated $\mathrm{S} / \mathrm{N}$ ratios (Fig. S5) for both sets of data are quite large (i.e. $>20)$, which ensures that the peak areas of the extracted ion peaks can be accurately and reproducibly quantified. This demonstrates that $\mathrm{MSH}$ and $\mathrm{CF}$ are both effective methods for exposing small, soluble proteins in non-scavenging buffers.

However, the exposure of complex, scavenging samples using the MSH endstation may require exposure times much greater than $30 \mathrm{~ms}$. The longer exposure times could potentially lead to a marked deterioration in MS data quality, making it difficult to quantify the unmodified and modified species of a particular peptide (Bohon et al., 2014; Gupta, Celestre et al., 2014; Gupta et al., 2007). Fig. S6 shows SDSPAGE gels of cytochrome $c$ samples exposed via MSH using progressively lower beam attenuations, or increased flux, while keeping exposure time constant. Sample degradation is apparent after the first time point in the samples receiving the higher X-ray doses as shown by the diffuse, barely visible bands on the gel. CF provides a way to deliver high X-ray doses to samples without sacrificing data quality because a small, high flux density beam can be used to deliver a high $\mathrm{X}$-ray dose to samples on a microsecond timescale, limiting sample degradation caused by secondary reactions.

To further compare the MSH and CF data obtained on XFP, protection factors (PFs) for each modified residue were calculated by dividing each residue's rate of modification (listed in Tables S2 and S3) by its intrinsic reactivity to hydroxyl radicals (Huang et al., 2015). The rate of modification is a function of both a side chain's solvent accessibility and intrinsic reactivity with hydroxyl radicals, so the calculation of PF values allows for the comparison of side chain solvent accessibility within a given protein on an absolute scale (Huang et al., 2015; Xu \& Chance, 2005). The PF values for the residues in each dataset were mapped onto the structure of rabbit cytochrome $c$ (Fig. 8) in order to create a visual representation of the regions within the protein that appear to be buried (i.e. less solvent accessible) or exposed (i.e. more solvent accessible). Both the MSH and CF data show a similar pattern of solvent accessibility with the majority of the observed modifications being highly solvent accessible, or located on the surface of cytochrome $c$.

Mapping the PFs onto the structure of rabbit cytochrome $c$ only provides a qualitative assessment of how well the calculated protection factors correspond to the computed solventaccessible surface area (SASA) of the amino acid side chains within the structure of cytochrome $c$, but a more quantitative measurement can be performed by determining the correlation coefficient for the scatter plot between $\log$ PF and the fractional SASA of the modified residues. The scatter plot for the MSH data is shown as an example and was found to have a correlation coefficient $(\rho)$ of -0.55 to $-0.58, p$-value $<0.05$ (Fig. 9), well within expectation based on previous studies (Huang et al., 2015). Thus, the $\rho$ obtained for our data establishes that our beamline setup and exposure parameters can provide accurate absolute information about the solvent accessibility of residue side chains within an intact, native protein.

\section{Summary and outlook}

NSLS-II XFP at 17-BM provides significantly higher flux density for XF studies than has previously been available, on track to exceed $500 \mathrm{~W} \mathrm{~mm}^{-2}\left(4.6 \times 10^{17}\right.$ photons s$\left.{ }^{-1} \mathrm{~mm}^{-2}\right)$ as NSLS-II reaches its full design current. Beam sizes from hundreds of micrometers to several millimeters, provided at two alternate endstation locations, allow XFP to adapt to multiple XF sample morphologies. Benchmarking experiments using a standard protein (cytochrome $c$ ) establish that accurate and reproducible in-solution XF structural data can be obtained using either the $\mathrm{CF}$ or MSH experimental endstations, providing users with flexibility in designing and conducting $\mathrm{XF}$ experiments. In the first year of the general 
A

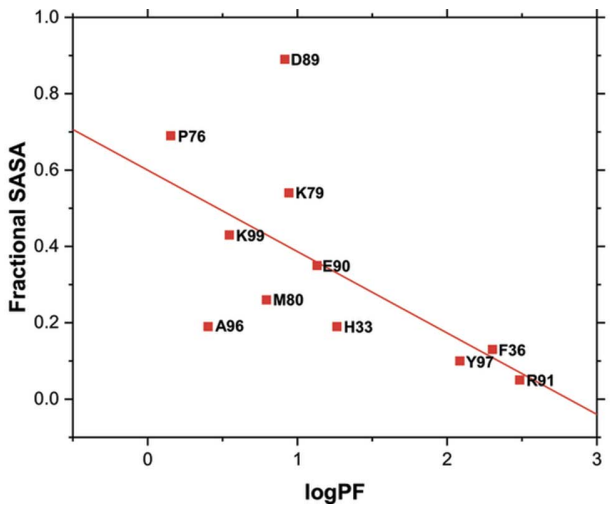

B

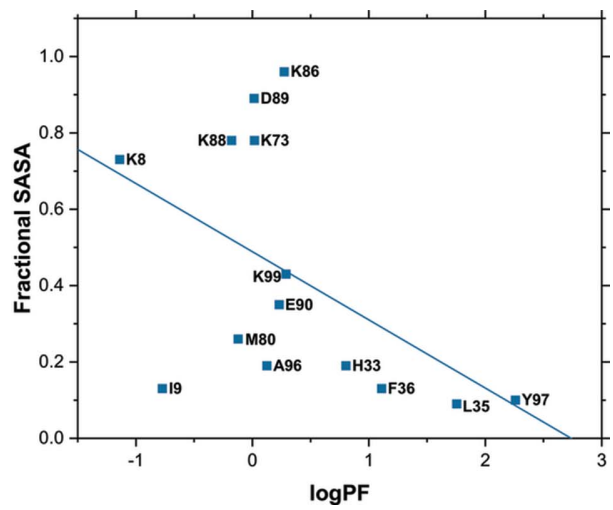

Figure 9

Representative scatter plots of log PF plotted against fractional SASA for the multi-sample holder (MSH) and capillary flow (CF) experimental data. The correlation coefficients for the CF (A) and MSH (B) data were $-0.58(p$-value $<0.05)$ and $-0.55(p$-value $<0.05)$, respectively.

user program at XFP, the beamline has already hosted $\mathrm{XF}$ experiments on viruses, live cells, organelles, protein complexes resistant to crystallization, pharmaceuticals binding with targets, peptide-nanoparticle interactions, prions ( $\mathrm{Li}$ et al., 2018), time-resolved studies (Du et al., 2019) and RNAprotein complexes, as well as more standard protein-protein interaction studies. Many of these studies were not feasible without the capabilities of XFP, and first science results are eagerly anticipated. We hope to encourage further innovation by optimizing sample delivery/exposure and adding complementary structural techniques to provide further insight into structure and function of biological macromolecules. Additional capabilities currently being developed at XFP include time-resolved footprinting apparatus and an ultra-highthroughput endstation that will allow users to expose 96 samples in as little as $4 \mathrm{~min}$. An additional endstation downstream of the XF endstations, currently intended for experiments requiring monochromatic radiation such as X-ray absorption spectroscopy, will be detailed in future work. NSLS-II XFP is the premier beamline for X-ray footprinting in the world, and we expect the expanded capabilities and continued upgrades of XFP endstations to enable significant contributions in the field of structural biology not possible with XF five to ten years ago. Potential users interested in performing $\mathrm{XF}$ experiments are encouraged to contact the corresponding authors to discuss the feasibility of their proposed experiments and to visit our webpage (https:// www.bnl.gov/nsls2/beamline/17-BM) to learn more about the requirements and process for submitting a general user proposal to NSLS-II.

\section{Acknowledgements}

The authors would like to thank the many NSLS-II staff involved in the partnership to construct and commission the XFP beamline. The 3PW spectrum was provided by $\mathrm{O}$. Chubar. The authors would like to thank E. Muller, M. Zou and J. Smedley for assistance with beam measurements. The authors would like to disclose that Mark Chance is a founder and shareholder of Neo Proteomics, which provides footprinting technology and services.

\section{Funding information}

The authors would like to thank Sydor Instruments (grant No. DE-SC0007482) for use of a quadrant beam monitor. Funding for beamline development was provided by the National Science Foundation, Division of Biological Infrastructure (grant No. 1228549; grant No. 1255340). This research used the 17-BM beamline of the National Synchrotron Light Source II, a US Department of Energy (DOE) Office of Science User Facility operated for the DOE Office of Science by Brookhaven National Laboratory under Contract No. DESC0012704. This publication was also made possible by the Center for Synchrotron Biosciences grant P30-EB-00998 from the National Institute of Biomedical Imaging and Bioengineering.

\section{References}

Adilakshmi, T., Bellur, D. L. \& Woodson, S. A. (2008). Nature, 455, 1268-1272.

Adilakshmi, T., Lease, R. A. \& Woodson, S. A. (2006). Nucleic Acids Res. 34, e64.

Arkilic, A., Allan, D. B., Caswell, T. A., Li, L., Lauer, K. \& Abeykoon, S. (2017). Synchrotron Radiat. News. 30(2), 44-45.

Baud, A., Aymé, L., Gonnet, F., Salard, I., Gohon, Y., Jolivet, P., Brodolin, K., Da Silva, P., Giuliani, A., Sclavi, B., Chardot, T., Mercère, P., Roblin, P. \& Daniel, R. (2017). J. Synchrotron Rad. 24, 576-585.

Bohon, J., D’Mello, R., Ralston, C., Gupta, S. \& Chance, M. R. (2014). J. Synchrotron Rad. 21, 24-31.

Bohon, J., Muller, E. \& Smedley, J. (2010). J. Synchrotron Rad. 17, 711-718.

Chubar, O., Rakitin, M. S., Chen-Wiegart, Y.-C., Chu, Y. S., Fluerasu, A., Hidas, D. \& Wiegart, L. (2017). Proc. SPIE, 10388, 1038811.

Clatterbuck Soper, S. F., Dator, R., Limbach, P. \& Woodson, S. (2013). Mol. Cell, 52, 506-516.

Du, Y., Duc, N. M., Rasmussen, S. G. F., Hilger, D., Kubiak, X., Wang, L., Bohon, J., Kim, H. R., Wegrecki, M., Asuru, A., Jeong, K. M., Lee, J., Chance, M. R., Lodowski, D. T., Kobilka, B. R., \& Chung, K. Y. (2019). Cell, 177, https:/doi.org/10.1016/j.cell.2019.04.022.

Gupta, S., Celestre, R., Petzold, C. J., Chance, M. R. \& Ralston, C. (2014). J. Synchrotron Rad. 21, 690-699.

Gupta, S., Chai, J., Cheng, J., D'Mello, R., Chance, M. R. \& Fu, D. (2014). Nature, 512, 101-104.

Gupta, S., D'Mello, R. \& Chance, M. R. (2012). Proc. Natl Acad. Sci. 109, 14882-14887. 
Gupta, S., Sullivan, M., Toomey, J., Kiselar, J. \& Chance, M. R. (2007). J. Synchrotron Rad. 14, 233-243.

Hao, Y., Bohon, J., Hulscher, R., Rappé, M. C., Gupta, S., Adilakshmi, T. \& Woodson, S. A. (2018). Curr. Protoc. Nucleic Acid Chem. 73, e52.

Huang, W., Peng, Y., Kiselar, J., Zhao, X., Albaqami, A., Mendez, D., Chen, Y., Chakravarthy, S., Gupta, S., Ralston, C., Kao, H.-Y., Chance, M. R. \& Yang, S. (2018). Nat. Commun. 9, 3520.

Huang, W., Ravikumar, K. M., Chance, M. R. \& Yang, S. (2015). Biophys. J. 108, 107-115.

Hulscher, R. M., Bohon, J., Rappé, M. C., Gupta, S., D’Mello, R., Sullivan, M., Ralston, C. Y., Chance, M. R. \& Woodson, S. A. (2016). Methods, 103, 49-56.

Kiselar, J. G., Janmey, P. A., Almo, S. C. \& Chance, M. R. (2003). Proc. Natl Acad. Sci. 100, 3942-3947.

Kiselar, J. G., Maleknia, S. D., Sullivan, M., Downard, K. M. \& Chance, M. R. (2002). Int. J. Radiat. Biol. 78, 101-114.

Li, Q., Wang, F., Xiao, X., Kim, C., Bohon, J., Kiselar, J., Safar, J. G., Ma, J. \& Surewicz, W. K. (2018). J. Biol. Chem. 293, 18494-18503.

Ralston, C. Y., Sclavi, B., Sullivan, M., Deras, M. L., Woodson, S. A., Chance, M. R. \& Brenowitz, M. (2000). Methods Enzymol. 317, 353-368.

Sangodkar, J., Perl, A., Tohme, R., Kiselar, J., Kastrinsky, D. B., Zaware, N., Izadmehr, S., Mazhar, S., Wiredja, D. D., O'Connor,
C. M., Hoon, D., Dhawan, N. S., Schlatzer, D., Yao, S., Leonard, D., Borczuk, A. C., Gokulrangan, G., Wang, L., Svenson, E., Farrington, C. C., Yuan, E., Avelar, R. A., Stachnik, A., Smith, B., Gidwani, V., Giannini, H. M., McQuaid, D., McClinch, K., Wang, Z., Levine, A. C., Sears, R. C., Chen, E. Y., Duan, Q., Datt, M., Haider, S., Ma'ayan, A., DiFeo, A., Sharma, N., Galsky, M. D., Brautigan, D. L., Ioannou, Y. A., Xu, W., Chance, M. R., Ohlmeyer, M. \& Narla, G. (2017). J. Clin. Invest. 127, 2081-2090.

Sclavi, B., Sullivan, M., Chance, M. R., Brenowitz, M. \& Woodson, S. A. (1998). Science, 279, 1940-1943.

Sclavi, B., Woodson, S., Sullivan, M., Chance, M. R. \& Brenowitz, M. (1997). J. Mol. Biol. 266, 144-159.

Sullivan, M. R., Rekhi, S., Bohon, J., Gupta, S., Abel, D., Toomey, J. \& Chance, M. R. (2008). Rev. Sci. Instrum. 79, 025101.

Takamoto, K. \& Chance, M. R. (2006). Annu. Rev. Biophys. Biomol. Struct. 35, 251-276.

Wang, L. \& Chance, M. R. (2017). Mol. Cell. Proteomics, 16, 706716.

Xu, G. \& Chance, M. R. (2005). Anal. Chem. 77, 4549-4555.

Xu, G. \& Chance, M. R. (2007). Chem. Rev. 107, 3514-3543.

Xu, H. \& Freitas, M. A. (2009). Proteomics, 9, 1548-1555.

Zhou, T., Ding, W., Gaowei, M., De Geronimo, G., Bohon, J., Smedley, J. \& Muller, E. (2015). J. Synchrotron Rad. 22, 13961402 . 